\title{
Stiffness test in lateral direction of temporary wooden building supports
}

\author{
Krzysztof Gromysz ${ }^{1, *}$ \\ ${ }^{1}$ Silesian University of Technology, Faculty of Civil Engineering, 44-100 Gliwice, ul. Akademicka 5, Poland
}

\begin{abstract}
Temporary supports consisting of a jack, a stack of wooden cubic elements and a iron plate are used during the removal of buildings deflections by uneven raising. Where the weight of a building is rested on temporary supports with a considerable length, unintentional displacements of buildings in the horizontal direction are seen. The displacements are connected with supports deformations caused by horizontal forces acting on the building part being raised. Non-vertically installed jacks, being part of the supports, are the most frequent reason for the occurrence of such forces. The jacks are not vertical due to deformations in the stack of wooden elements, upon which they are rested. In such case, the stiffness of temporary supports is essential for the safety of the deflection removal process. Laboratory tests of temporary supports were carried out and they showed that their stiffness, understood as a horizontal force divided by a displacement in the acting direction of the force, is not constant. The stiffness of supports is decreasing as the displacement amplitude grows. A considerable decrease in supports stiffness was experienced when positive longitudinal deformations occurred in the cross section of the support elements. As a result, the unconnected elements of the supports were unable to transmit positive stresses of this number. For the investigated range of loads, the deformations of the material of the supports elements were elastic. Inelastic forces were however generated along the contact points of the elements forming part of the supports, and such forces were responsible for creating a hysteresis loop and energy dissipation by the supports. The system, when a full load-unload cycle was applied, was returning to the initial position. Higher values of the energy dissipation coefficient correspond to higher values of a displacement amplitude.
\end{abstract}

\section{Introduction}

Building deflections are removed (straightened) by uneven raising of structures with hydraulic jacks (Fig. 1) [1]. The jacks of length $l_{\text {ja }}$ are installed in openings made in advance in structure walls (Fig. 2a). The straightening process is comprised of three phases. Force is triggered in all jacks in the first phase, as a result of which the building is detached (Fig. 2a). Horizontal rupture of a building, running between the jacks, is then formed. A horizontal detachment plane, dividing a structure to the underground part and the straightened part, is then created. An identical displacement is applied in all the jacks in the second phase, as a result of which a building part located over the detachment plane is raised to a height of $20 \mathrm{~mm}$ to $30 \mathrm{~mm}$ (Fig. 2b). This is necessary so that, in the third phase of straightening, i.e. uneven raising, the part being straightened and this remaining in the ground do not hook up. In the last phase of straightening, hydraulic jacks, due to limited piston extension of $220 \mathrm{~mm}$, need to be underlain periodically. Cubic wooden elements with the cross-section $(b / h)$ of $400 / 300 \mathrm{~mm}$ and a thickness of 100 $\mathrm{mm}$ are mounted underneath the jacks for buildings with up to two floors. Such elements are forming stacks whose length $l_{\mathrm{wo}}$ depends on the structure raising height. A $20 \mathrm{~mm}$ thick steel plate (pl. 400/200/20) has to be installed between a jack and a stack to limit the values of local stresses transmitted from the jack base onto stacks of wooden elements. Temporary supports of buildings are formed this way, onto which the entire weight of the part of the building being straightened is placed. The length of such supports ( $l_{\text {sup }}-$ Fig. $\left.2 c\right)$ depends on the raising height. If deflections of considerably deflected buildings [3, 4] or multisegments buildings are removed, $l_{\text {sup }}$ reaches up to $2 \mathrm{~m}$.

Unintentional vertical and horizontal displacements of buildings are witnessed during deflection removal in the event where a building is placed on temporary supports with a considerable length. Displacements in the vertical direction are the result of changes in the length of supports. The changes are caused by temporary and cyclic loads of stacks of wooden elements (the underpinning) by jacks. This issue was a subject of the laboratory tests discussed in [2].

Corresponding author: krzysztof.gromysz@polsl.pl 


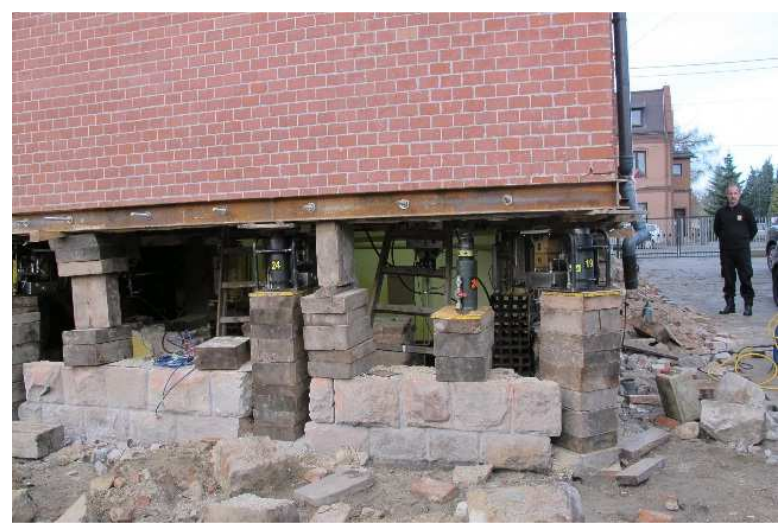

Fig. 1. Example of wooden supports installed in the wall of the straightened building

a)

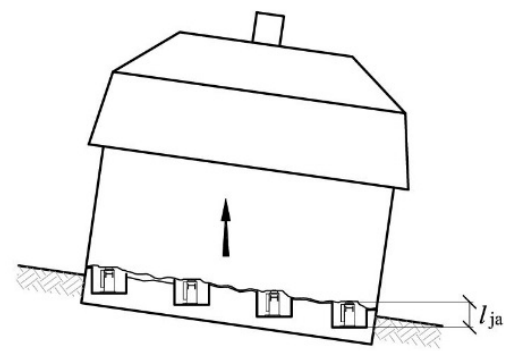

b)

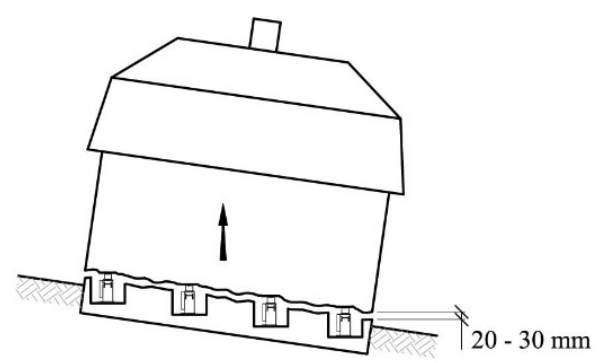

c)

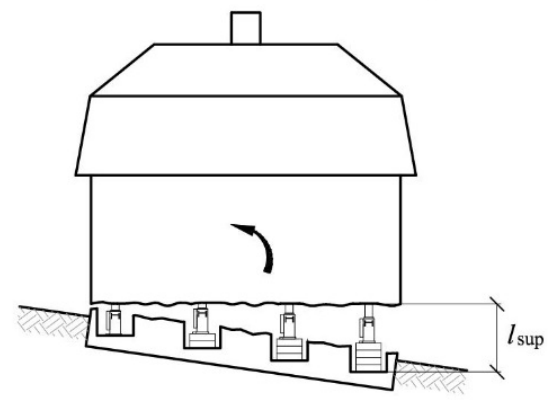

Fig. 2. Building straightening process: a), b), c) phases of straightening (description in the text)

Displacements in the horizontal direction occur because supports transmit a horizontal force designated as $Q_{x}$. This force may be caused by wind acting on the part of the building being raised, by forces of inertia and by exceptional loads. However, the most common cause of the force $Q_{x}$ are non-vertically installed jacks generating the horizontal force $Q_{x}$. The lack of verticality in the position of jacks results from a second-order deformation of temporary supports with the length $l_{\text {sup }}$ and from considerable values of the vertical forces $Q_{z}$ (structure weight) applied to such supports. The non-vertical installation of jacks also derives from rotation $\theta$ of the temporary support base positioned on the part of the building remaining in the ground (Fig. 3).

The presence of displacements in the horizontal direction is dangerous because this may lead to the loss of stability of a single support, and in an extreme situation to the loss of stability of the entire building being straightened and to a constructional disaster. The height $l_{\text {sup }}$ of temporary supports is limited to prevent this. This is achieved by dividing the uneven raising of the building into several stages. A straightening process has to be interrupted several times as a result, temporary supports have to be dismounted and walls have to be constructed for the part of the building remaining in the ground.

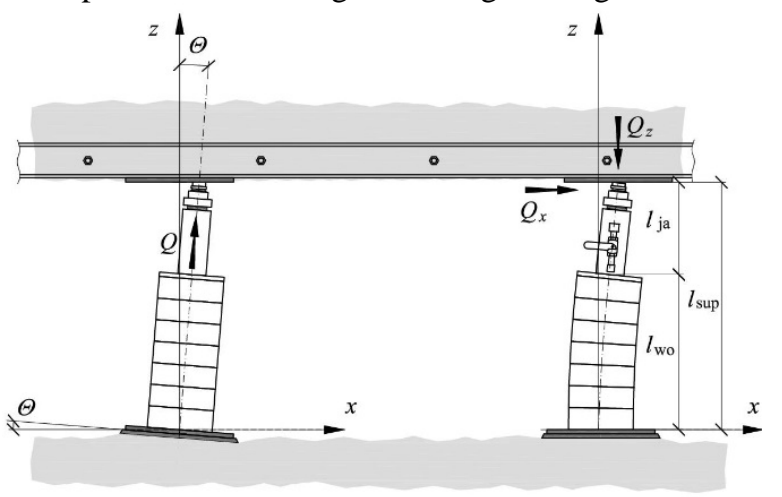

Fig. 3. Rotation $\theta$ of the temporary support (the support on the left) as the cause for existence of the horizontal load $Q_{x}$ of the support with the length $l_{\text {sup }}$ (the support on the right)

The analyzes of eccentrically loaded elements have been carried out mainly in relation to reinforced concrete elements [5], steel columns [6] and masonry structures [7, 8]. Respectively, the results of the experimental research concern mainly GFRP Reinforced Geopolymer Concrete [9], steel [6] and masonry structures [10-12]. However, the possibility of transferring eccentrically applied forces by temporary supports consisting of a stack of elements has not been studied. Meanwhile, the possibility of transferring an eccentrically applied load by these supports determines the safety of the building deflection removal process.

The stiffness of supports in the horizontal direction has to be known in order to analyse, through calculations, the horizontal displacements of a structure placed on temporary supports. Tests have been designed and carried out for this purpose, the aim of which was to determine the stiffness of temporary supports of buildings in the horizontal direction.

\section{Programme of tests}

As outlined above, parameters of temporary supports had to be investigated because it was necessary to analyse, in calculations, the horizontal displacements of structures resting on temporary supports. Supports' parameters, such as the dependencies describing stiffness of supports, have to be defined in modern computer software. For this reason, the non-linear stiffness of a support according to its displacement is to be described as a result of the undertaken tests. 
It was taken into account, when developing the programme of tests, that the upper part of the support is a jack piston head provided with a joint. It was also assumed that the support base, resting on the wall of the building part remaining below the detachment surface, cannot move or rotate $(\theta=0-$ Fig. 3$)$. In the case where the resultant of the longitudinal force $Q_{z}$ is situated in the core of the cross-section $\left(e_{\mathrm{d}}<h / 6-\right.$ Fig. $\left.4 \mathrm{a}\right)$, a static scheme of a temporary support is, therefore, represented by a support with the length $l_{\text {sup }}$, loaded with the vertical force $Q_{z}$ and with the horizontal force $Q_{x}$ (Fig. 4a). It is practically not possible to duplicate such a scheme of a single temporary support in laboratory conditions, with the values of the longitudinal force $Q_{z}$ reaching up to $200 \mathrm{kN}$. Another test scheme for a temporary support was used for this reason. It is a freely supported element, with the length $l=2 l_{\text {sup, }}$, loaded with two forces: $Q_{x, l / 2}$ perpendicular to the axis of the element applied in the midspan and axial $Q_{z}$ applied at the and (Fig. 4b). A deformed axis of this element, in which a shearing force with the value $Q_{x}$ occurs, corresponds to a composition of an axis of two deformed supports working as a support, loaded with forces with the value $Q_{x}$. The studied element, with the length $l$, is, therefore, a composition of two elements with the length $l_{\text {sup }}$, and a centre of symmetry of the studied system is running through its midspan. In particular, the stiffnesses of the systems from Figures 4a (a support with the length $l_{\text {sup }}$ ) and $4 \mathrm{~b}$ (a beam with the length $2 l_{\text {sup }}$ ) are equal to each other, in case where the forces $Q_{x}$ and $Q_{z}$ with the same values exist there. This results from the fact that the quotient of the load in $x$ direction in relation to the displacement $u_{x, l / 2}$, in the systems from Figures $4 \mathrm{a}$ and $4 \mathrm{~b}$, are equal.

a)

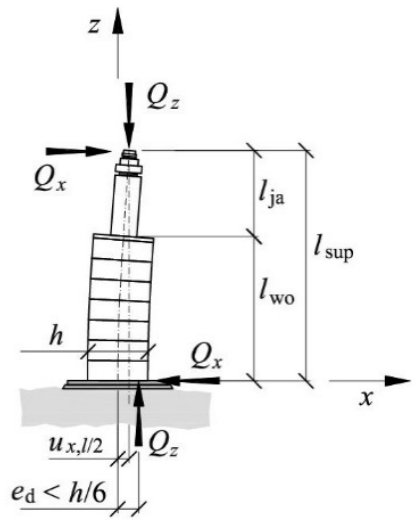

b)

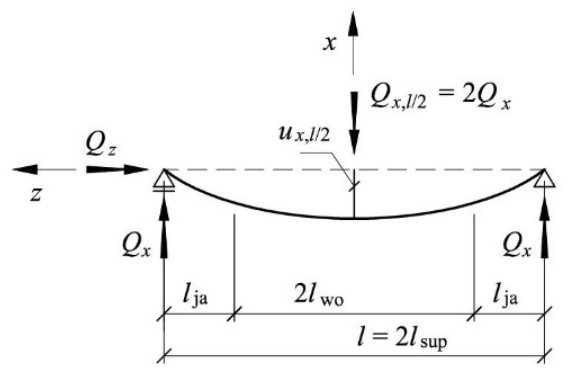

Fig. 4. Scheme of: a) temporary support, b) tested element

Laboratory tests were designed for a system with the length $2 l_{\text {sup }}$ set in a horizontal position. The studied system consists of two piston jacks placed in the direction of the axis $z$, with their bases positioned towards each other, triggering the force $Q_{z}$ imitating the building weight (fig. 5). A stack of fourteen rectangular elements made of oak wood was placed between the jacks. A dimension of each wooden element in the direction of the axis $x$ is $h=300$ $\mathrm{mm}$, in the direction $y$ it is $b=400 \mathrm{~mm}$ (Fig. 5) and in the direction of the axis $z$ it is $100 \mathrm{~mm}$. The direction of growth rings of all the wooden elements was coinciding with the direction of the axis $y .20 \mathrm{~mm}$ thick steel plates (pl. 300/400/20 mm) are situated between the bases of the jacks and a stack of wooden elements. The system was mounted in a stand (Fig. 5) consisting of two heads in the form of plate girders with a height of $440 \mathrm{~mm}$. The heads were connected with two ties, and each tie consists of two [ 200 sections. Dynamometers measuring the force $Q_{z}$ were mounted between the plate girders and the jacks heads.
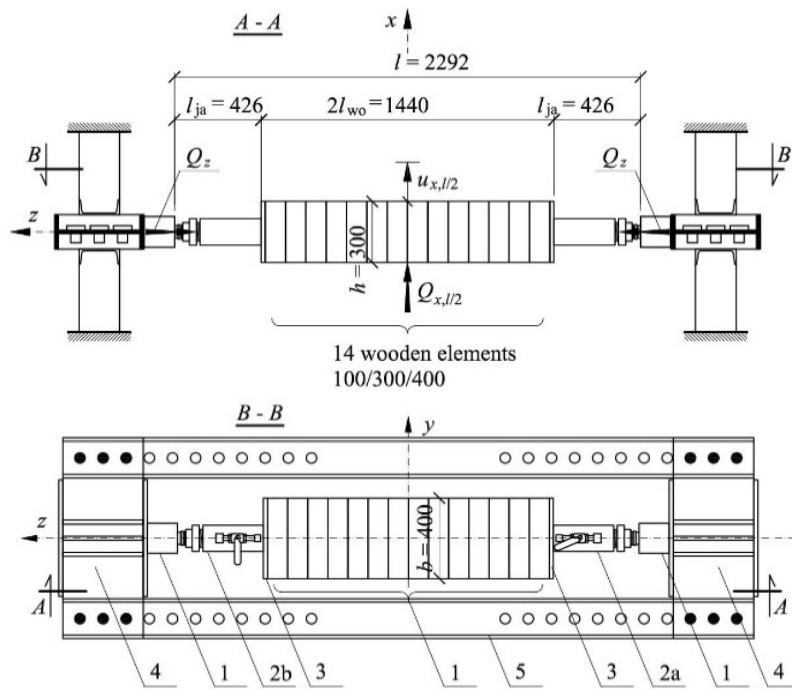

Fig. 5. The studied element and the test stand (positive senses of the load $Q_{x, l / 2}$ and displacements $u_{x, l / 2}$ are provided in the Figure): 1 - stack of fourteen rectangular wooden elements, each with dimensions of 300/400/100 mm, $2 \mathrm{a}$ - active hydraulic jack, $2 \mathrm{~b}-$ inactive hydraulic jack, 3 - steel plate with dimensions of $300 / 400 / 20 \mathrm{~mm}, 4$ - the head of the stand, 5 - ties

The tests consisted of producing the constant force $Q_{z}$, while at the same time forcing the displacements $u_{x, / / 2}$ in the system midspan. The displacements were forced cyclically up (positive value) and down (negative value) by applying a force with the value $Q_{x, l / 2}$ in the direction $x$. Two constant values of the axial force $Q_{z}$ (force acting towards the axis $z$ ) were assumed in the tests, accounting for $100 \mathrm{kN}$ and $200 \mathrm{kN}$, and two values of the displacement amplitude of $10 \mathrm{~mm}$ and $60 \mathrm{~mm}$. It was planned to perform four tests, as listed in Table 1 . The material designation of rectangular elements is given in the title of each test (wo - wood), along with the length $l_{\text {wo }}$ of the stack of wooden elements $(1440 \mathrm{~mm})$ and information about the value of the force $Q_{z}(100 \mathrm{kN}$ or 200 $\mathrm{kN}$ ) and about the assumed displacement amplitude $u_{x, l / 2}$ (10 $\mathrm{mm}$ or $60 \mathrm{~mm})$. 
Table 1. Designation of tests.

\begin{tabular}{|c|c|c|}
\hline Test designation & $\begin{array}{c}Q_{z} \\
\mathrm{kN}\end{array}$ & $\begin{array}{c}\text { Displacement } \\
\text { amplitude } u_{x, l / 2}, \\
\mathrm{~mm}\end{array}$ \\
\hline Wo. $1440 / 100 / 10$ & 100 & 10 \\
\hline Wo. $1440 / 200 / 10$ & 200 & 10 \\
\hline Wo. $1440 / 100 / 60$ & 100 & 60 \\
\hline Wo. $1440 / 200 / 60$ & 200 & 60 \\
\hline
\end{tabular}

\section{Progress and results of tests}

The tests were performed according to the programme presented in the previous section. The vertical force $Q_{x, l / 2}$, forcing the displacements $u_{x, l / 2}$, was applied with two single-acting hydraulic jacks (Fig. 6a). The jack forcing upward displacements (a positive sense of the displacement $u_{x, l / 2}$ ) was positioned below the tested element on a slab of large forces, and the jack forcing downward displacements (a negative sense of the displacement $u_{x, l / 2}$ ) was above the tested element and was mounted to a steel frame (Fig. 6b). The values of the forces were measured with the accuracy of $0.001 \mathrm{kN}$. Displacements were measured with the accuracy of 0.001 $\mathrm{mm}$ with induction sensors. The vertical force $Q_{x, / 2}$ was applied with the speed of about $0.1 \mathrm{kN}$ per second, which is similar to the conditions existing when eliminating deflections of real structures. The measured values of the forces and displacements were recorded with the frequency of two readings per second.

a)

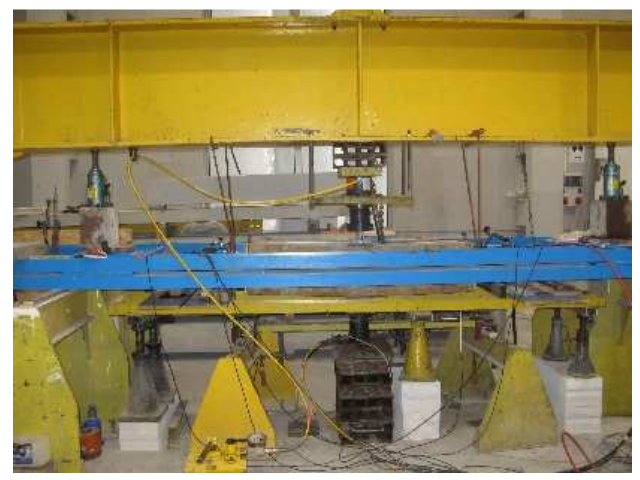

b)

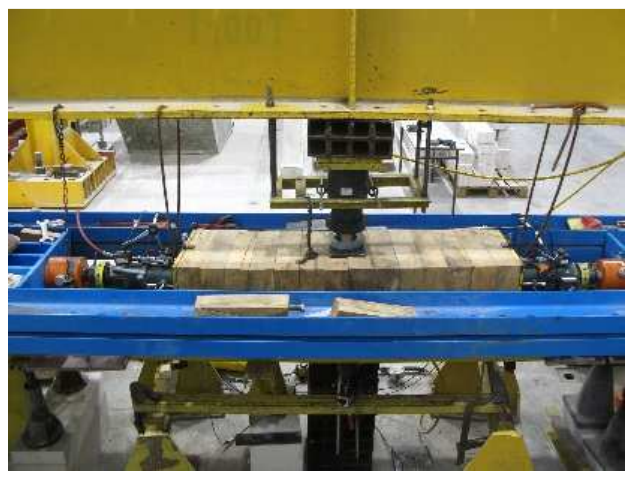

Fig. 6. Stand during the tests
Upward and downward displacements of the system were forced alternately for each test, thus obtaining four hysteresis loops. Hysteresis loops corresponding to the last cycle were only presented below for all the tests. Each cycle is characterised by two extreme values of displacements, i.e. $u_{x, l / 2, \text { min }}-\mathrm{a}$ displacement with a negative value (directed downwards) and $u_{x, l / 2, \max }-\mathrm{a}$ displacement with a positive value (directed upwards). The load values $Q_{x, l / 2, \min }$ and $Q_{x, l / 2, \max }$ correspond to such displacements (Fig. 7). The obtained values of extreme forces and displacements are given in Table 2.

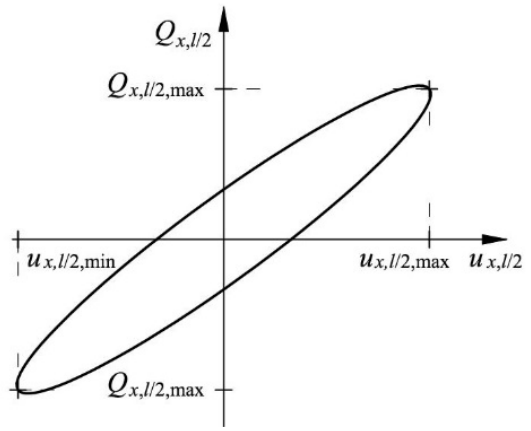

Fig. 7. Hysteresis loop with the characteristic values of displacement and loads marked.

The obtained load-displacement $\left(Q_{x, l / 2}-u_{x, l / 2}\right)$ hysteresis loops are presented graphically in Figures 8 11. Top branches were distinguished on such loops, corresponding to the case where the load value $Q_{x, l / 2}$ is growing and the system is displaced upwards, and a bottom branch, corresponding to the case where the load value $Q_{x, l / 2}$ is falling and the system is displaced downwards.

Table 2. Extreme values of the load force $Q_{x, l / 2}$ and the corresponding values of the displacements $u_{x, l / 2}$.

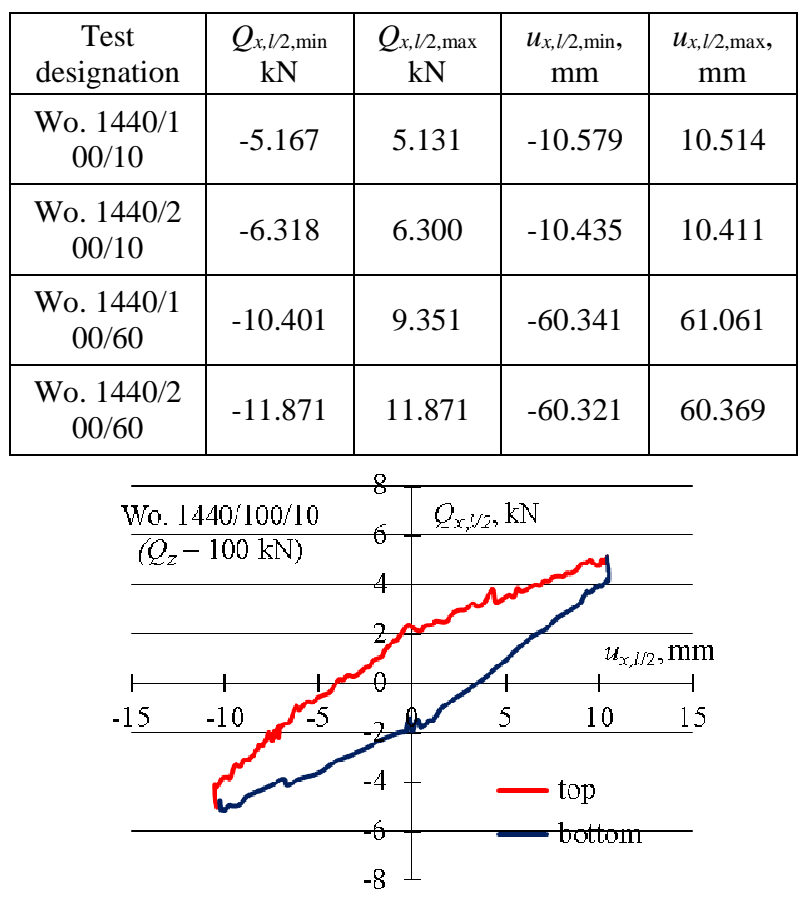

Fig. 8. Wo. 1440/100/10 test results 


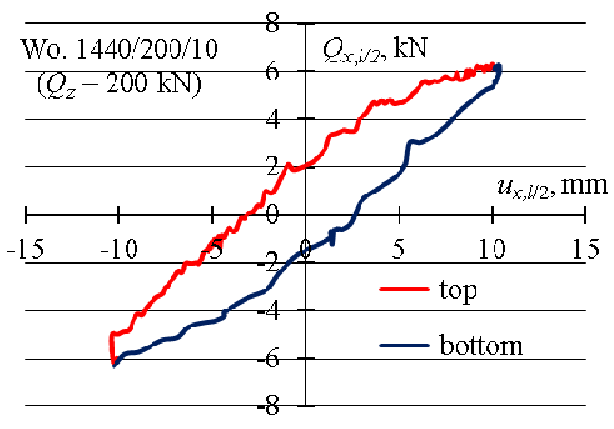

Fig. 9. Wo. 1440/200/10 test results

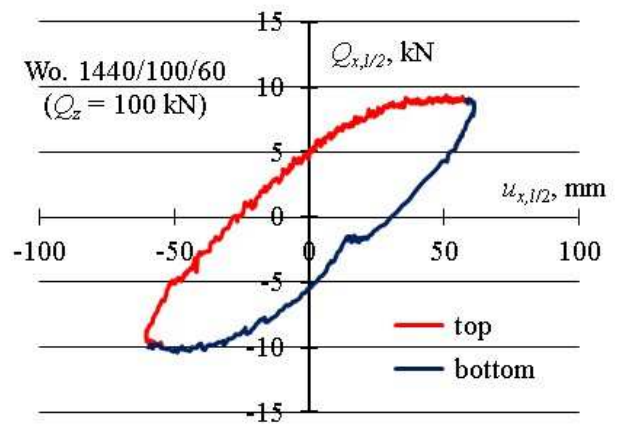

Fig. 10. Wo. 1440/100/60 test results

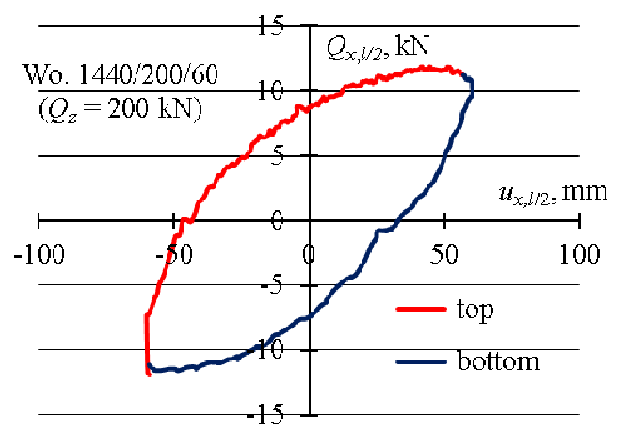

Fig. 11. Wo. 1440/200/60 test results

\section{Analysis of tests results}

An analysis of tests results was performed to determine the stiffness of supports and their inelastic parameters.

\subsection{Supports stiffness}

The following method was employed for determination of the stiffness of supports in the direction $x$. First, the polynomials describing the load-displacement dependencies for the distinguished two branches of the hysteresis loops (top, bottom) were determined with the method of least squares. Functions describing the stiffness of temporary supports, as derivatives of respective polynomials, were then calculated.

A satisfactory description of the dependency $Q_{x, l / 2}\left(u_{x, l / 2}\right)$ for all the tests and loads was obtained with a polynomial of second degree. The values of the determination coefficients $R^{2}$ of approximation of the bottom and top branches of the hysteresis loop were not smaller than 0.990 , denoting that the tests results were well described. Such a high value of $R^{2}$ results from the fact that the material of the elements of the examined supports experienced only elastic deformations. The polynomials $Q_{x, l / 2}\left(u_{x, l / 2}\right)$, determined independently for the top and bottom branch, were listed in Table 3 for all the tests with the corresponding values $R^{2}$. The polynomials describing the dependencies $Q_{x, l / 2}\left(u_{x, l / 2}\right)$, determined for successive measurements, were presented graphically on the charts given in Figures 12a, 13a, 14a, 15a. According to the adopted procedure, the support stiffness in the direction corresponding to a given load phase was determined as a derivative of the polynomial:

$$
k_{x}=\frac{\mathrm{d} Q_{x, l / 2}\left(u_{x, l / 2}\right)}{\mathrm{d} u_{x, l / 2}} .
$$

The expressions on stiffness, calculated this way, were compiled in the respective rows of Table 3.

Table 3. Polynomials approximating the dependencies

$Q_{x, l / 2}\left(u_{x, l / 2}\right)$ and the derivatives of such polynomials.

\begin{tabular}{|c|c|c|}
\hline \multirow{2}{*}{$\begin{array}{c}\text { Designa } \\
\text { tion }\end{array}$} & \multirow{2}{*}{ Branch } & $Q_{x, l / 2}\left(u_{x, l / 2}\right) ; R^{2}$ \\
\hline & & $\frac{\mathrm{d} Q_{x, l / 2}}{\mathrm{~d} u_{x, l / 2}}=k_{x}$ \\
\hline \multirow{4}{*}{$\begin{array}{c}\text { Wo. } \\
1440 / 10 \\
0 / 10\end{array}$} & \multirow{2}{*}{ top } & $\begin{array}{c}Q_{x, l / 2}\left(u_{x, l / 2}\right)=-0.0158 u_{x, l / 2}^{2}+ \\
0.4389 u_{x, l / 2}+1.9728 ; R^{2}=0.996\end{array}$ \\
\hline & & $k_{x}=-0.0316 u_{x, l / 2}+0.4389$ \\
\hline & \multirow{2}{*}{ bottom } & $\begin{array}{c}Q_{x, l / 2}\left(u_{x, l / 2}\right)=0.0133 u_{x, l / 2}^{2}+ \\
0.4507 u_{x, l / 2}-1.7175 ; R^{2}=0.998\end{array}$ \\
\hline & & $k_{x}=0.0266 u_{x, l / 2}+0.4507$ \\
\hline \multirow{4}{*}{$\begin{array}{c}\text { Wo. } \\
1440 / 20 \\
0 / 10\end{array}$} & \multirow{2}{*}{ top } & $\begin{array}{l}Q_{x, l / 2}\left(u_{x, l / 2}\right)=-0.0152 u_{x, l / 2}^{2}+ \\
0.5860 u_{x, l / 2}+2.1024 ; R^{2}=0.996\end{array}$ \\
\hline & & $k_{x}=-0.0304 u_{x, l / 2}+0.5860$ \\
\hline & \multirow{2}{*}{ bottom } & $\begin{array}{l}Q_{x, l / 2}\left(u_{x, l / 2}\right)=0.0144 u_{x, l / 2}^{2}+ \\
0.5946 u_{x, l / 2}-1.6541 ; R^{2}=0.997\end{array}$ \\
\hline & & $k_{x}=0.0288 u_{x, l / 2}+0.5946$ \\
\hline \multirow{4}{*}{$\begin{array}{c}\text { Wo. } \\
1440 / 10 \\
0 / 60\end{array}$} & \multirow{2}{*}{ top } & $\begin{array}{c}Q_{x, l / 2}\left(u_{x, l / 2}\right)=-0.0014 u_{x, l / 2}^{2}+ \\
0.1499 u_{x, l / 2}+5.1622 ; R^{2}=0.995\end{array}$ \\
\hline & & $k_{x}=-0.0028 u_{x, l / 2}+0.1499$ \\
\hline & \multirow{2}{*}{ bottom } & $\begin{array}{l}Q_{x, l / 2}\left(u_{x, l / 2}\right)=0.0011 u_{x, l / 2}^{2}+ \\
0.1567 u_{x, l / 2}-5.0690 ; R^{2}=0.991\end{array}$ \\
\hline & & $k_{x}=0.0022 u_{x, l / 2}+0.1567$ \\
\hline \multirow{4}{*}{$\begin{array}{l}\text { Wo. } \\
1440 / 20 \\
0 / 60\end{array}$} & \multirow{2}{*}{ top } & $\begin{array}{c}Q_{x, l / 2}\left(u_{x, l / 2}\right)=-0.0022 u_{x, l / 2}^{2}+ \\
0.1521 u_{x, l / 2}+9.1698 ; R^{2}=0.990\end{array}$ \\
\hline & & $k_{x}=-0.0044 u_{x, l / 2}+0.1521$ \\
\hline & \multirow{2}{*}{ bottom } & $\begin{array}{l}Q_{x, l / 2}\left(u_{x, l / 2}\right)=0.0020 u_{x, l / 2}^{2}+ \\
0.1252 u_{x, l / 2}-7.4077 ; R^{2}=0.993\end{array}$ \\
\hline & & $k_{x}=0.0040 u_{x, l / 2}+0.1252$ \\
\hline
\end{tabular}


It was found that the stiffness of supports depends on the displacement value $u_{x, l / 2}$ and on the load phase. For the top branch, the support has maximum stiffness for the displacement of $u_{x, l / 2, \min }$. Next, as the displacement value is growing, stiffness is falling, reaching the smallest value for a limit displacement of $u_{x, l / 2, \max }$.

For the bottom branch, the support has maximum stiffness for the extreme displacement of $u_{x, l / 2, \max .}$ Next, as the displacement value is falling, the support stiffness is falling, reaching the smallest value in the position of $u_{x, l / 2, \min }$.

Average stiffnesses for the investigated range of displacements of $u_{x, l / 2, \min }$ to $u_{x, l / 2, \max }$ were also determined based on the tests results. Such stiffnesses were called diagonal stiffnesses, designated as $k_{x \text {,diag, }}$ and calculated from the dependency:

$$
k_{x, \text { diag }}=\frac{Q_{x, l / 2, \max }-Q_{x, l / 2, \min }}{u_{x, l / 2, \max }-u_{x, l / 2, \min }} .
$$

Charts showing values of the stiffnesses $k_{x}$, corresponding to top and bottom branches in particular

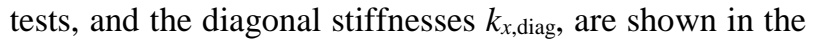
charts given in Figures 12b, 13b, 14b, $15 b$.

It can be concluded by analysing the charts $k_{x}\left(u_{x, l / 2}\right)$ that the values of diagonal stiffnesses $k_{x \text {,diag }}$ are approximately equal to stiffnesses $k_{x}$ determined for a zero displacement value of $u_{x, l / 2}$. It can also be noticed that support stiffness depends not only on the range of the investigated displacements, but also on the value of the force $Q_{z}$. Within the investigated range, the higher values of the load $Q_{z}$ correspond to higher support stiffness. The obtained $k_{x, \text { diag }}$ values are listed in Table 4.

a)

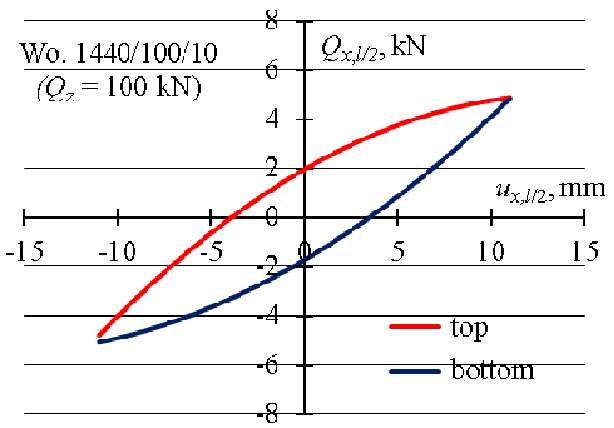

b)

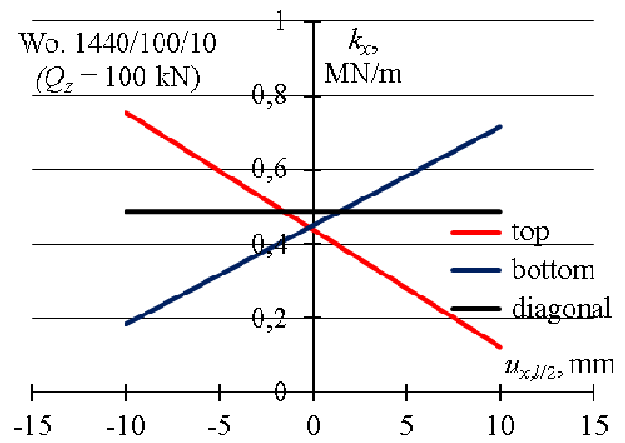

Fig. 12 Wo. test results 1440/100/10: a) approximation of the top and bottom branch of the hysteresis loop $Q_{x, 1 / 2}-u_{x, l / 2}$ with polynomials, b) stiffnesses corresponding to the top and bottom branch determined on the basis of (1) and diagonal stiffness determined according to (2) a)

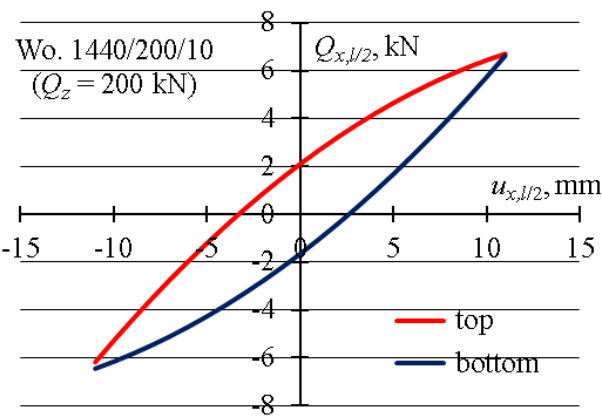

b)

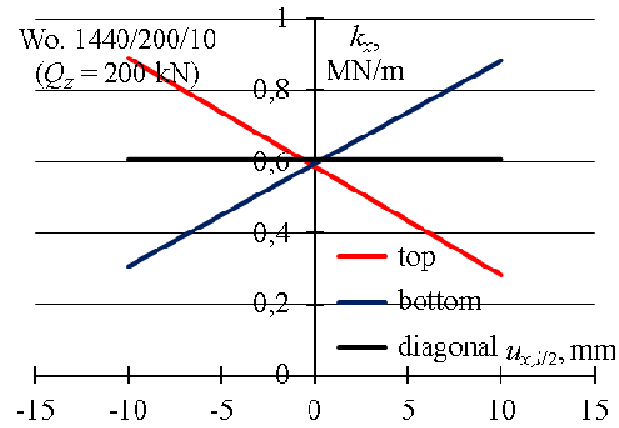

Fig. 13. Wo. 1440/200/10 test results : a) approximation of the top and bottom branch of the hysteresis loop $Q_{x, l / 2}-u_{x, l / 2}$ with polynomials, b) stiffnesses corresponding to the top and bottom branch determined on the basis of (1) and diagonal stiffness determined according to (2)

a)

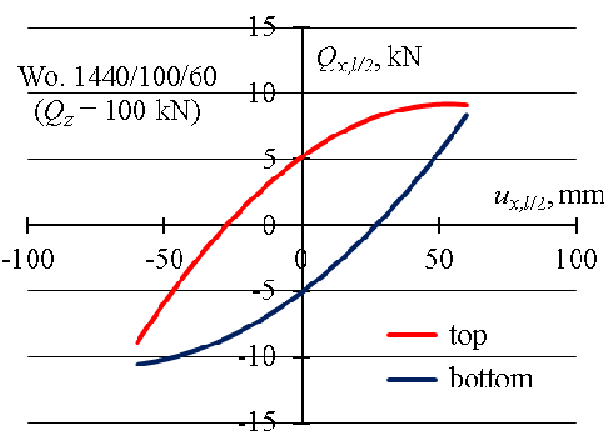

b)

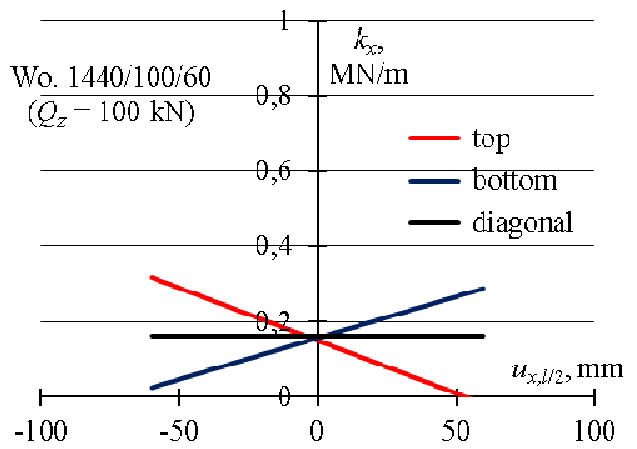

Fig. 14. Wo. 1440/100/60 test results : a) approximation of the top and bottom branch of the hysteresis loop $Q_{x, l / 2}-u_{x, l / 2}$ with polynomials, b) stiffnesses corresponding to the top and bottom branch determined on the basis of (1) and diagonal stiffness determined according to (2) 
a)

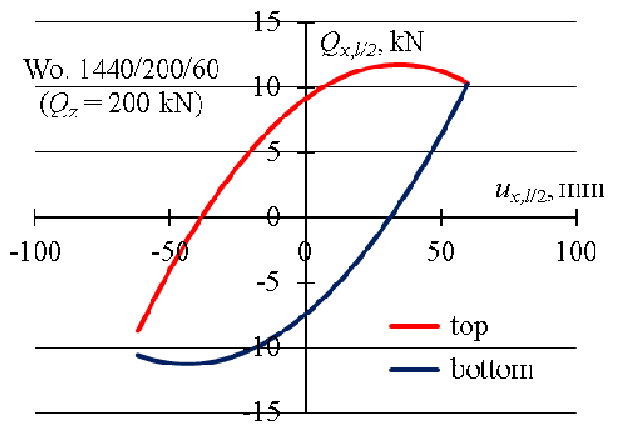

b)

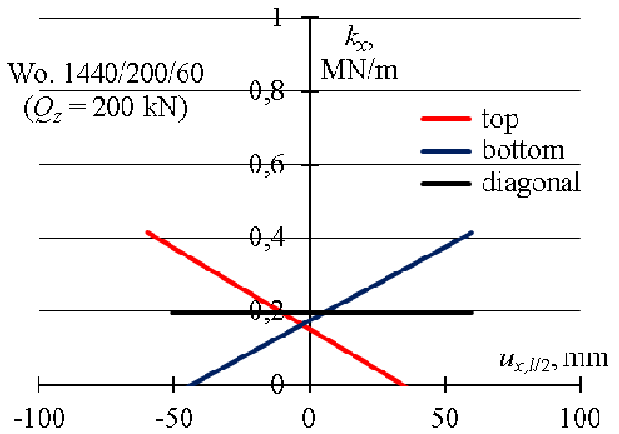

Fig. 15. Wo. 1440/200/60 test results: a) approximation of the top and bottom branch of the hysteresis loop $Q_{x, l / 2}-u_{x, l / 2}$ with polynomials, b) stiffnesses corresponding to the top and bottom branch determined on the basis of (1) and diagonal stiffness determined according to (2)

A list provided in Table 4 shows that much higher stiffness is seen for a support with a displacement amplitude of $10 \mathrm{~mm}$. The above results from the fact that, for this displacement amplitude, the value of normal stresses $\left(\sigma_{z, l / 2}\right)$ in all cross-sections of the support had always a negative value. The values of such stresses in the element midspan in Wo. 1440/100/10 and Wo. 1440/200/10 tests were determined from the dependency (3) and were provided in Table 4.

$$
\begin{aligned}
& \sigma_{z, l / 2, \max / \min } \\
& =-\frac{Q_{z}}{A} \pm \frac{0.25 l\left|Q_{x, l / 2, \min }\right|+Q_{z}\left|u_{x, l / 2, \min }\right|}{W}
\end{aligned}
$$

where $A=b \cdot h, W=b \cdot h^{2} / 6$.

It should be noted that for tests where the displacement amplitude $u_{x, l / 2}$ was $60 \mathrm{~mm}$, the formula (3) could not be applied. Deformations with a positive sens, which could not be transmitted by the unconnected wooden elements, occurred in such tests.

Table 4. The calculated stiffness values $\left(k_{x, \text { diag }}\right)$ and extreme

\begin{tabular}{|c|c|c|}
\hline \multirow{2}{*}{ Test designation } & \multirow{2}{*}{$\begin{array}{c}k_{x, \text { diag }} \\
\mathrm{MN} / \mathrm{m}\end{array}$} & $\sigma_{z, l / 2, \max }, \mathrm{MPa}$ \\
\hline & & $\sigma_{z, l / 2, \min }, \mathrm{MPa}$ \\
\hline \multirow{2}{*}{ Wo. $1440 / 100 / 10$} & \multirow{2}{*}{0.488} & -0.164 \\
\hline & & -1.503 \\
\hline \multirow{2}{*}{ Wo. $1440 / 200 / 10$} & \multirow{2}{*}{0.605} & -0.715 \\
\hline & & -2.618 \\
\hline Wo. $1440 / 100 / 60$ & 0.163 & $\begin{array}{c}\text { Not } \\
\text { determined }\end{array}$ \\
\hline Wo. $1440 / 200 / 60$ & 0.197 & $\begin{array}{c}\text { Not } \\
\text { determined }\end{array}$ \\
\hline
\end{tabular}
values of normal stresses in a stack of cubic elements

\subsection{Inelastic parameters of supports}

If the load line does not coincide with the unload line, and if the top and bottom branch of the hysteresis loop exist, this signifies the occurrence of non-conservative forces in the tested system. Such forces, in a load-unload cycle, perform the work whose value equals the area of the hysteresis loop. The work of non-conservative forces, performed on the deformations of the tested element, was designated as $\chi$. An area hatched horizontally in Figure 16 is an indicator of dissipated energy. A formula for a polygon area was used for calculating its area, when the coordinates of vertexes are known (detail in Fig. 16).

$$
\chi=\frac{1}{2} \sum_{i=2}^{n} Q_{i, x, l / 2}\left(u_{i+1, x, l / 2}-u_{i-1, x, l / 2}\right) .
$$

The value of potential energy of the investigated element for a maximum deformation is designated by $E_{\mathrm{p}}$. This energy is calculated as the field of the area hatched vertically in Figure $16 . E_{\mathrm{p}}$ was hence estimated with the dependency:

$$
E_{\mathrm{p}}=\frac{1}{2} k_{x, \text { diag }}\left(u_{x, l / 2, \min }\right)^{2} .
$$

The energy dissipation coefficient value of the system was defined as the quotient:

$$
\psi=\frac{\chi}{E_{\mathrm{p}}} .
$$

The values $\chi, E_{\mathrm{p}}$ and $\psi$, obtained in particular tests, are listed in Table 5 . The energy dissipation coefficient value $\psi$ for the tests conducted ranges between 0.505 and 1.017. Higher values of this coefficient correspond to higher values of a displacement amplitude. On the other hand, the effect of the value of the force $Q_{z}$ on the value of the energy dissipation coefficient $\psi$ is not unequivocal. For the tests where the amplitude $u_{x, l / 2}$ was $10 \mathrm{~mm}$, the increase of the force $Q_{z}$ from $100 \mathrm{kN}$ to $200 \mathrm{kN}$ led to a small decrease of the value $\psi$ from 0.505 to 0.431 . However, for the tests where the amplitude $u_{x, l / 2}$ was 10 $\mathrm{mm}$, the increase of the value of the force $Q_{z}$ from $100 \mathrm{kN}$ to $200 \mathrm{kN}$ led to a significant decrease of the value $\psi$ from 0.758 to 1.017 .

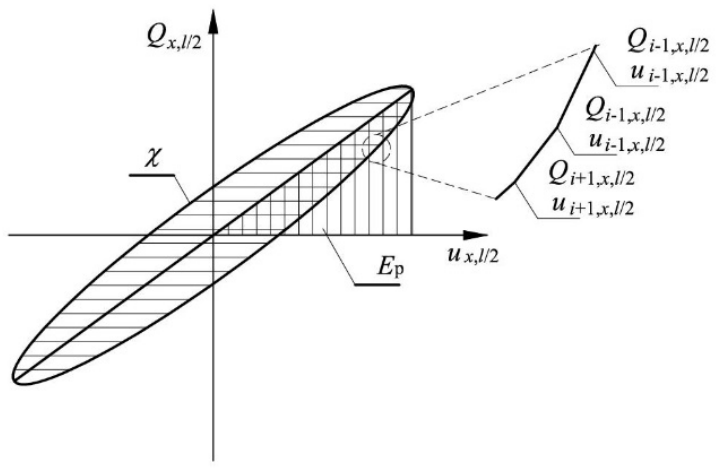

Fig. 16. The designations adopted: $E_{\mathrm{p}}$ - potential energy (vertically hatched area), $\chi$ - area of hysteresis loop (horizontally hatched area) 
Table 5. The calculated values of potential energy $\left(E_{\mathrm{p}}\right)$, of the area of hysteresis loop $(\chi)$ and of the energy absorption coefficient $(\psi)$.

\begin{tabular}{|c|c|c|c|}
\hline Test designation & $\begin{array}{c}E_{\mathrm{p},} \\
\mathrm{N} \cdot \mathrm{m}\end{array}$ & $\begin{array}{c}\chi, \\
\mathrm{N} \cdot \mathrm{m}\end{array}$ & $\begin{array}{c}\psi, \\
-\end{array}$ \\
\hline Wo. $1440 / 100 / 10$ & 26.985 & 54.530 & 0.505 \\
\hline Wo. $1440 / 200 / 10$ & 32.804 & 56.582 & 0.431 \\
\hline Wo. $1440 / 100 / 60$ & 303.307 & 919.039 & 0.758 \\
\hline Wo. $1440 / 200 / 60$ & 358.448 & 1458.844 & 1.017 \\
\hline
\end{tabular}

The results obtained, concerning the investigated elements, lead to the following findings. The deformations of the material of the supports elements are elastic. The system, after performing a full load-unload cycle, returns to the initial position and no permanent displacements are observed. Inelastic forces are however produced in the system, which exist along the contact points of the elements forming part of the supports. Under the growing load, with small displacement values, in the surrounding of the position $u_{x, l / 2}=0$, an approximately linear dependency exists between the change of the load $Q_{x, l / 2}$ and the change of the displacement $u_{x, l / 2}$. This denotes that the deformations in the material of the supports elements and the displacements connected with their mutual pressing are growing proportionally. A considerable decrease in supports stiffness occurs when positive deformations occur in the cross section of the support elements. As a result, the unconnected elements of the stack were unable to transmit stresses with such number. This was experienced, for the tests conducted, for the displacement $u_{x, l / 2}$ of about $12 \mathrm{~mm}$. Higher support stiffnesses are seen at the beginning of the unloading process, which means that no displacements take place, practically, along the contact points of the elements forming part of the supports and only their material is deformed. As the load is increasing again, support stiffness is falling, which is linked to a mutual displacement of the support elements.

\section{Summary}

It was demonstrated with the laboratory tests performed that the stiffness of temporary supports of buildings is not constant. A considerable decrease in support stiffness is encountered when positive deformations occur in the cross section of the support elements. As a result, the unconnected elements of the stack are unable to transmit stresses with such number. The deformations of the material of the supports elements are elastic. The system, after applying a full load-unload cycle, returns to the initial position and no permanent displacements are observed. Inelastic forces are however produced in the system, which exist along the contact points of the elements forming part of the supports. Such forces are responsible for creating a hysteresis loop and for existence of energy dissipation. Higher values of the energy dissipation coefficient correspond to higher values of a displacement amplitude.

\section{References}

1. K. Gromysz, Methods of Removing Buildings Deflection Used In Poland. IOP Conf. Ser.: Mater. Sci. Eng. 245032096 (2017).

2. K. Gromysz, Deformations of temporary wooden supports used to reduce building deflections in mining areas. E3S Web of Conferences 36, 03002 (2018).

3. M. Wyleżoł, Determination of measurable deflection of buildings in the aspect of their designing. Collective work edited by $K$. Gromysz and $R$. Domagata. Outline of selected issues of Civil Engineering. Publishing House of the Silesian University of Technology: 191-198 (2017).

4. M. Wyleżoł, Measurement methods of building structures deflections. E3S Web of Conferences 36, 02010 (2018)

5. M.Y. Kabir, E. Shafei, Plasticity modelling of FRPconfined circular reinforced concrete columns subjected to eccentric axial loading. Composites: Part B 43 (2012) 3497-3506.

6. S.-D. Nie, S.-B. Kang, L. Shen, B. Yang, Experimental and numerical study on global buckling of Q460GJ steel box columns under eccentric compression. Engineering Structures 142 (2017) 211-222.

7. E. Bernat-Maso, L. Gil, P. Roca, Numerical analysis of the load-bearing capacity of brick masonry walls strengthened with textile reinforced mortar and subjected to eccentric compressive loading. Engineering Structures 91 (2015) 96-111.

8. E. Moradabadi, D.F. Laefer, J.A. Clarke, P.B. Lourenço, A semi-random field finite element method to predict the maximum eccentric compressive load for masonry prisms. Construction and Building Materials 77 (2015) 489-500.

9. M. Elchalakania, A. Karrecha, M. Dongao, M.S.M. Alib, B. Yangc, Experiments and Finite Element Analysis of GFRP Reinforced Geopolymer Concrete Rectangular Columns Subjected to Concentric and Eccentric Axial Loading. Structures 14 (2018) 273289.

10. E. Bernat-Maso, L. Gil, C. Escrig, Analysis of brick masonry walls strengthened with fibre reinforced polymers and subjected to eccentric compressive loads. Construction and Building Materials 84 (2015) 169-183.

11. L. Cavaleri, A. Failla, L.L. Mendola, M. Papia, Experimental and analytical response of masonry elements under eccentric vertical loads. Engineering Structures 27 (2005) 1175-1184.

12. E. Bernat, L. Gil, P. Roca, C. Escrig, Experimental and analytical study of TRM strengthened brickwork walls under eccentric compressive loading. Construction and Building Materials 44 (2013) 35-47. 